\title{
EL UNIVERSO EN LA URDIMBRE DEL ESPACIO/TIEMPO
}

The universe in the space/time warp

\author{
Aldo Vivar Mendoza ${ }^{1}$
}

\begin{abstract}
Resumen
En su Teoría General de la Relatividad, Albert Einstein propuso la existencia de ondas gravitacionales como curvaturas del espacio/ tiempo. Rainer Weiss, Kip Thorne y Barry Barish, por casi cuarenta años, concibieron y desarrollaron el interferómetro de láser como instrumento para detectar tales ondas que fueron descubiertas en 2015, hallazgo que les valió el premio Nobel de Física 2017.
\end{abstract}

Palabras claves: Premio Nobel, Física, ondas, gravedad.

Abstract

In his General Theory of Relativty, Albert Einstein introduced the term Gravitational Waves as ripples in the space/time. Rainer Weiss, Kip Thorne and Barry Barish embraced this idea and worked for almost 40 years to develop a suitable detector, the laser interferometer. The experimental discovery of gravitational waves in 2015 deserved them the Nobel Prize in Physics 2017.

Keywords: Nobel Prize, physics, waves, gravity.

F a película Contacto basada en el libro homónimo del astrofísico Carl Sagan ofrece un ejemplo de aquellos centinelas que pacientemente esperan recibir una señal desde el espacio interestelar. En la película, circa 1990, los científicos reciben una señal codificada en números primos que llega desde la estrella Vega a 25 años luz de la Tierra, señal que es interpretada como expresión de vida inteligente que viene del espacio estelar. Sin embargo, luego de varios intentos de decodificación los científicos se dan cuenta que la señal es el discurso de bienvenida de Adolf

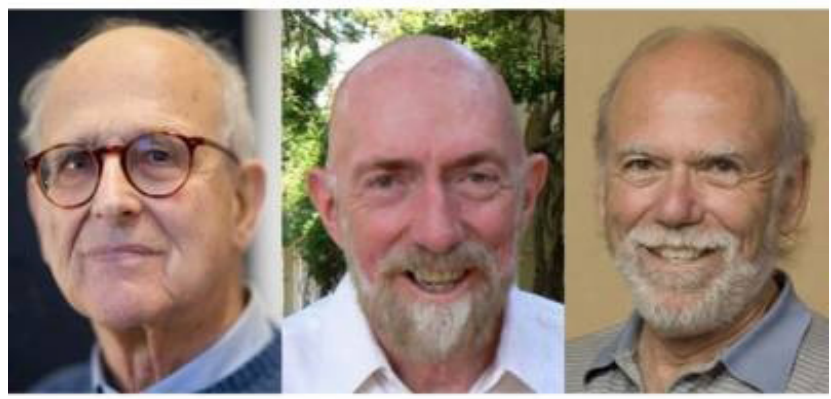

Rainer Weiss, Kip Thorne y Barry Barish, laureados con el Premio Nobel de física 2017. (Foto: MiT/Keenan Pepper/R. Hahn)

Hitler en los Juegos Olímpicos de Berlín en 1936 que en la vida real fue la primera señal de televisión que salió de la atmósfera terrestre. Estas señales habían retornado a la Tierra más de sesenta años después rebotada desde una estrella tan lejana.

Para entender el Nobel de Física de este año se debe encontrar la analogía con Contacto, la existencia de centinelas que con paciencia esperan señales del espacio exterior. En el caso del Nobel 2017 nos remitimos a LIGO (Laser Interferometer Gravitational Wave Observatory), un telescopio particular no diseñado para detectar luz o radiación electromagnética proveniente del espacio

\footnotetext{
${ }^{1}$ Médico internista. Hospital Nacional Arzobispo Loayza. Universidad Peruana Cayetano Heredia.
} 
sino para escuchar el sonido interestelar del movimiento del universo; es decir, LIGO ha sido diseñado para detectar las ondas del espacio/tiempo.

Las ondas gravitacionales son pliegues del espacio/ tiempo que se propagan a la velocidad de la luz y fueron descritas matemáticamente por Albert Einstein en 1915 como parte de la Teoría de la Relatividad. Einstein propuso que el tiempo y el espacio son maleables y conforman una cuarta dimensión: el espacio/ tiempo, un continuo donde está inmerso el universo entero, un concepto muy distinto al que propuso Newton, el éter inmaterial por donde se desplazan la luz y los cuerpos celestes suspendidos por medio de la fuerza de la gravedad.Años antes Maxwellhabía enunciado el concepto de ondas electromagnéticas que se desplazaban por el universo y que actuaban como fuerzas de energía que acompañaban a la de la gravedad.

Para Einstein, el espacio/tiempo es una urdimbre que comprende todo el universo que cuando se curva genera la fuerza de la gravedad, cuando la curvatura es extrema se generan los agujeros negros. Dentro de esta arquitectura celestial, el espacio/tiempo presenta depresiones provocadas por grandes masas algo parecidas a la huella que deja el cuerpo en una cama o pelotas que discurren sobre una manta. Los planetas y la luz van a alterar su movimiento por el efecto de enormes masas sobre el espacio/tiempo que vibra y genera ondas gravitacionales cuando una gran masa se acelera, cuando una estrella explota o cuando dos agujeros negros rotan entre sí. Tales ondas gravitacionales se expanden como el sonido en una forma muy parecida a las ondas que se generan cuando una pequeña piedra cae sobre agua quieta.
Einstein, al igual que con su concepto de los agujeros negros, tenía dudas de su real existencia y pensó acaso que tan solo eran la ilusión de una ecuación matemática; más aún, dudaba de que alguna vez fueran medidas debido al tiempo tan corto de su duración, unos escasos milisegundos. Así como para la teoría de la relatividad la gravedad es una curvatura del espacio/tiempo, algo difícil de pensar ya que no se es capaz de interpretar lo que no se conoce, se necesitaba de mucha imaginación para asumir la idea de las ondas gravitacionales y de cómo medirlas para los parámetros tridimensionales de la vida tal como la conocemos.

La primera evidencia indirecta vino en los años 1970's a través de un radiotelescopio que midió la energía perdida de dos pulsares que rotaban entre sí. La cantidad de esta energía disipada encajaba perfectamente con los cálculos teóricos estimados para las ondas gravitacionales, y por este descubrimiento Joseph Taylor y Russell Hulse obtuvieron el premio Nobel de Física en 1993 ("por el descubrimiento del pulsar y las nuevas posibilidades que se abren en el estudio de la gravitación"). También por los años 1970's, Kip Thorne, un físico teórico trabajando en el California Institute of Technology (Caltech) y Rainer Weiss, del Massachussets Institute of Technology (MIT), simultáneamente realizaron predicciones teóricas acerca de la posibilidad real de medir las ondas gravitacionales pero para ello debían de inventar y diseñar nuevos equipos no disponibles entonces. Tales ideas se basaron en teorías y experiencias previas.

En1957, uncongresosobreondasgravitacionales realizado en Pittsburg gatilló los trabajos de Joseph Weber de la Universidad de Maryland, quien diseñó una antena que funcionaba como un diapasón (horquilla) para detectar 
el sonido. Su trabajo fue publicado en 1960 y logró detectar radiaciones gravitacionales pero sus hallazgos no fueron replicados por otros investigadores y se consideró un error técnico. Pero el concepto quedó y esta basado en la propiedad de interferencia de la luz que hace capaz la medición de distancias. El instrumento de medición es el interferómetro de Michelson, una fuente que emite un rayo de luz que es partido en dos haces perpendiculares entre sí y que son reflejados por un espejo en el extremo distal de los brazos dispuestos en L, al llegar al mismo tiempo al punto del detector se anulan entre sí y no se detecta ninguna señal.

Por separado, en Caltech y en MIT, Thorne y Weiss, desde mediados de los años 1970's, trabajaron prototipos de sensores utilizando rayos láser que medían distancias de centímetros a pocos metros en el espacio interestelar. Durante estas pruebas identificaron las posibles interferencias del ruido de fondo: sismos, gradientes térmicas dentro de los tubos al vacío, inestabilidad del láser, tráfico pesado de la ciudad, etc. A medida que el tiempo pasaba la tecnología fabricaba mejores y más grandes aparatos de emisión láser. Ronald Drever en Glasgow fabricó un prototipo de 10 metros.

Pronto se dieron cuenta de la necesidad de construir sensores más grandes y para ello requerían un mayor presupuesto así que Thorne y Weiss postularon, a inicios de los 1980 's, a la National Science Foundation (NSF) para el financiamiento de sus proyectos. La NSF decidió financiar un solo proyecto trabajado en forma conjunta entre Caltech y MIT. De este modo, Thorne y Weiss se embarcaron junto a Ronald Drever (quien falleció el pasado marzo) a diseñar un sensor que eliminara el ruido interestelar de fondo que "ensucia" la medición de las ondas gravitacionales. Encontraron la fórmula diseñando un gigantesco interferómetro, un sistema de espejos y un emisor de rayos láser con dos tubos perpendiculares (que se disponen en $\mathrm{L}$ ). Si la señal es un ruido de fondo -que llega en una onda simétrica- el sistema de espejos la enfrenta a la vez y la anula. Si fuera una onda gravitacional -que viene en espiral- al afectar primero un brazo de la L y luego al otro no será anulada y será medida. Cada brazo del LIGO tenía una longitud de $4 \mathrm{~km}$ y se construyeron dos observatorios LIGO idénticos separados por $3000 \mathrm{~km}$, uno en Louisiana y otro en el estado de Washington. Siguiendo con las analogías es posible imaginar dos hilos tensados en forma perpendicular colocados sobre la superficie de un estanque que reciben la onda vibratoria producto de una piedra que cae al agua.

La mayor dificultad fue el fabricar equipos de muy alta precisión que detectaran este sonido que duraría una fracción de milisegundo, lo que para el espacio interestelar es como el piar de un ave al atardecer, así de repentino y efímero. Weiss y Thorne demoraron cerca de 40 años en crear los equipos, tan sensibles y delicados y a la vez resistentes pues deben soportar las inclemencias de la naturaleza terrestre. Estos investigadores inventaron un nuevo tipo de rayo láser, nuevos materiales y diseñaron la construcción de inmensos tubos al vacío. En 1994 se unió Barry Barish como líder del proyecto quien asumió el reto como una empresa de cooperación global convocando a miles de investigadores alrededor del mundo. El grupo original estuvo compuesto solo de 40 científicos. Esto aceleró notablemente el desarrollo del proyecto y 1994 marca la fecha de inicio efectiva del LIGO.

El 14 de setiembre del 2015, pocos días después de entrar oficialmente en funciones, los dos observatorios LIGO detectaron las primeras señales de la onda gravitacional que 


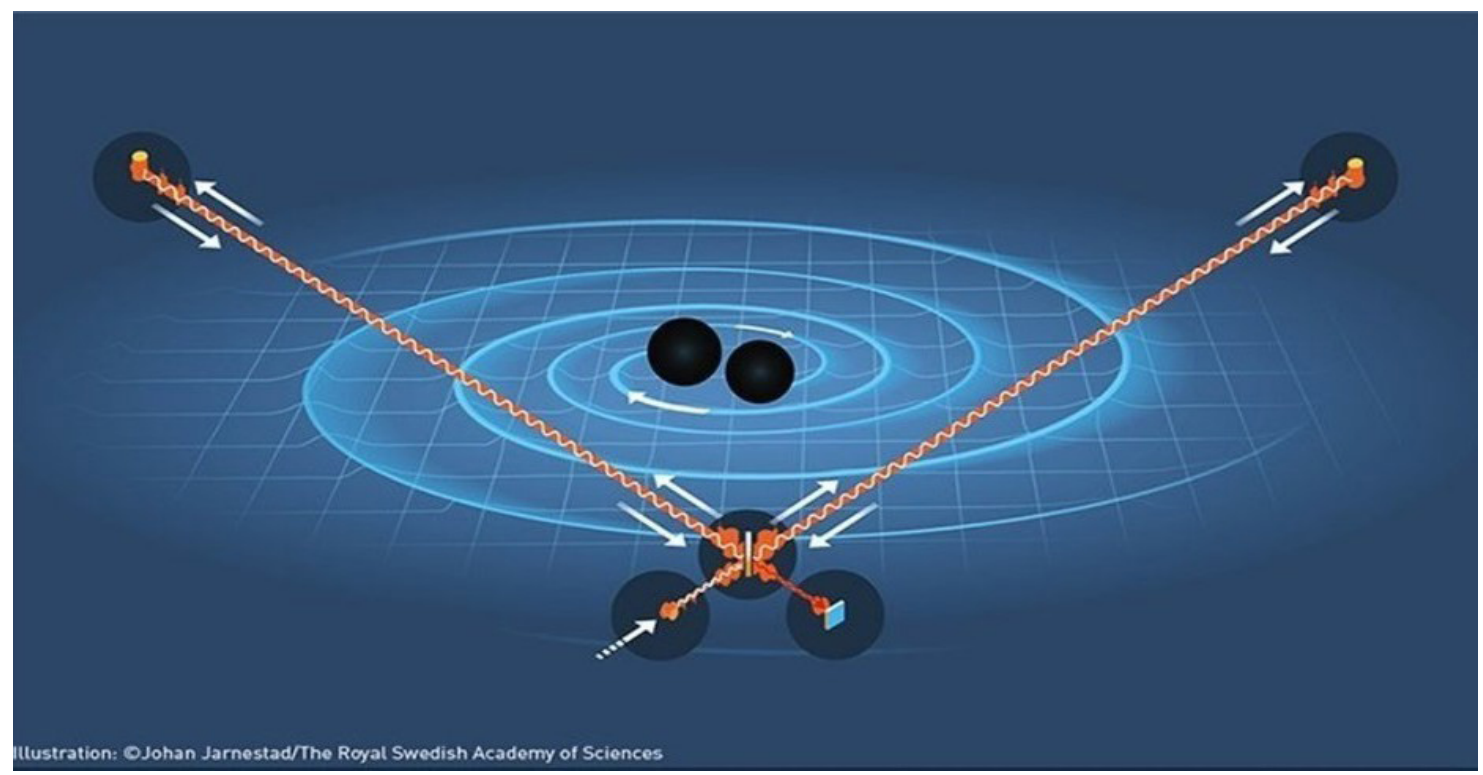

Ilustración: Johan JARnestad/The Royal Swedish ACADEMy of ScIEnces

duraba tan solo unas décimas de segundo. El análisis reveló que la onda era producto de una colisión de dos agujeros negros que se orbitaban mutuamente a la velocidad de la luz, colisionando y fusionándose con una energía de la magnitud de 62 soles juntos, una colisión que se calculó había ocurrido 1300 millones de años, el sonido de un instante infinitesimal en la vida del universo, el canto del cisne de una gigantesca colisión cósmica.

Thorne, Weiss y Barish, ganadores del premio Nobel de Física 2017, no revelaron este hallazgo inmediatamente -ni siquiera a sus esposas-, y sometieron los datos a una serie de pruebas para asegurarse que fuera real y no producto del ruido de fondo o del azar, calcularon incluso de que si este ruido fuera por azar su chance era de $2 \times 10^{-7}$, es decir de una probabilidad de 0.0000002 . Recién develaron el secreto, a sus familias y al mundo, en febrero del 2017. En agosto del mismo año, la estación gemela VIRGO en Europa (la localidad de Cascina, cerca de Pisa) confirmó los resultados. El espacio/tiempo no era producto de una fórmula matemática, ya se tenía evidencia directa pues era capaz de ser medido.

Eisntein estaría satisfecho, el espacio/tiempo existe como se sugiere en la película Interestelar de Christopher Nolan, la aventura de un viajero que alcanzando la velocidad de la luz es capaz de recorrer distancias inimaginables, volver a su pasado aprovechando la curvatura del espacio/tiempo y sus atajos -los llamados agujeros de gusano- y volver a la Tierra casi de la misma edad de la que salió para reencontrarse con su hija que dejó de niña y verla muy anciana y cercana a la muerte pues viajando a la velocidad de la luz se envejece mucho menos. Interestelar es una fábula del amor filial, el egoísmo humano que atraviesa el universo a través de la teoría de la relatividad, una película que tuvo la asesoría científica de Kip Thorne, uno de los Nobel ya mencionados. Es queen el espacio/tiempo nada es casualidad.

\section{Bibliografía Recomendada}

1. Hawking S, Modinov L. The Grand Design. Bantan 2012. 
2. Hawking S. The Illustrated On the Shoulder of Giants. Running Press, 2004.

3. March R. Física para poetas. Siglo XXI editores, 1977.

4. Rovelli C. Siete breves lecciones de Física. Anagrama, 2016.

5. Scientific background: The laser interferometer gravitational-wave observatory and the first direct observation of gravitational waves. URL disponible en: https: / / www.nobelprize.org / nobel_ prizes / physics / laureates / 2017 / advanced. html

6. Farr. B., Schelbert GM, Trouille L. Gravitational wave science in the high classroom. Am J Phys. 2012; 80: 898. 